\title{
Emotion Recognition Using Wearables: A Systematic Literature Review - Work-in-progress
}

\author{
Stanisław Saganowski ${ }^{1,2, *}$, Anna Dutkowiak ${ }^{1}$, Adam Dziadek ${ }^{3}$, Maciej Dzieżyc ${ }^{1,2}$, Joanna Komoszyńska ${ }^{1}$ \\ Weronika Michalska ${ }^{1}$, Adam Polak ${ }^{4}$, Michał Ujma ${ }^{3}$, Przemysław Kazienko ${ }^{1,2}$ \\ ${ }^{I}$ Department of Computational Intelligence, Wroctaw University of Science and Technology, Wrocław, Poland \\ ${ }^{2}$ Faculty of Computer Science and Management, Wrocław University of Science and Technology, Wrocław, Poland \\ ${ }^{3}$ Capgemini, Wroctaw, Poland \\ ${ }^{4}$ Faculty of Electronics, Wrocław University of Science and Technology, Wrocław, Poland \\ "stanislaw.saganowski@pwr.edu.pl
}

\begin{abstract}
Wearables like smartwatches or wrist bands equipped with pervasive sensors enable us to monitor our physiological signals. In this study, we address the question whether they can help us to recognize our emotions in our everyday life for ubiquitous computing. Using the systematic literature review, we identified crucial research steps and discussed the main limitations and problems in the domain.

Index Terms - emotion, emotion recognition, affective computing, wearable, smartwatch, systematic literature review, survey, review, smart device, smart band, personal device
\end{abstract}

\section{INTRODUCTION}

Emotions drive most of our decisions [1], not only intuitive ones [2], so they directly affect our everyday life. Most of the research on emotion recognition conducted so far focused on participant (subject) reactions evoked by the prepared stimuli in the controlled environment (laboratory setup). Therefore, complex emotion identification in the real-life environment, especially for pervasive computing, remains a significant challenge for this relatively new but promising field of study.

It is quite difficult to provide a commonly agreed definition of emotion. We should rather consider a set of features that distinguish an emotion from a non-emotion [3]. Overall, affect is seen as a neurophysiological state that is consciously accessible but not directed at any specific entity. Mood is a lasting and not very intense sensation. Finally, a short, intense, and directed feeling is described as emotion [4]. Further in this review, we refer to emotions interchangeably with affect. Besides, it is clear that two affective computing topics: stress and emotion recognition have recently become separate research lines [5]. Emotions can be identified and evaluated from different but complementary points of view:

1) Subjective perception of the participant (self-assessment of the subject),

2) Reaction of participant's organism (physiological signals), which is objective but may be contaminated by the

This work was partially supported by the National Science Centre, Poland, project no. 2016/21/B/ST6/01463; European Union's Horizon 2020 research and innovation program under the Marie Skłodowska-Curie grant agreement No. 691152 (RENOIR); the Polish Ministry of Science and Higher Education fund for supporting internationally co-financed projects in 2016-2019 no $3628 / \mathrm{H} 2020 / 2016 / 2$; and the statutory funds of the Department of Computational Intelligence, Wroclaw University of Science and Technology. individual's body condition and functioning (influenced by drugs or illnesses),

3) Behavioral signals like facial expressions, voice, specific body movements or even keystroke patterns [6],

4) External evaluation made by the subject's peers, e.g. an adult recognizing the state of the child [7].

In this paper, we focus on the second perspective - physiological signals that can be gathered using pervasive sensors built into wearable devices like smartwatches, wrist bands, smart rings or headbands. These kinds of devices possess an undoubted advantage. Due to their unobtrusiveness and convenience, they facilitate pervasive computing and contextaware systems by monitoring human affective states in the real-life environment, a.k.a field studies.

Recently, survey studies on emotion recognition have changed their primary focus from the EEG-based solutions [8], [9], through facial and speech analysis [10], [11], to physiology-oriented [4], [12]. In [12], all essential aspects of emotion identification (emotion models, stimuli, features, classifiers, etc.) are described, but it does not consider whether the research was conducted in the lab or field setup. Schmidt at al. respect the environment of the surveyed studies and conclude that emotion recognition outside the controlled lab is much more difficult [4]. The authors mention about 50 stress and emotion recognition papers (15 of which were field or constrained field studies).

We go a step further and consider only studies that are (or could be) placed in the field, i.e. they use devices and techniques that allow us to conduct the study in everyday situations. Additionally, by following the systematic literature review (SLR) procedure, we cover all relevant research published up to date. We expect SLR to be completed by the date of the conference. It means the extended results will be presented during the workshop.

\section{Systematic Literature RevieW}

A great method to summarize existing knowledge in a given domain is Systematic Literature Review (SLR). It involves identifying, evaluating and interpreting available research relevant to a certain research question [13]. In our SLR, we are posing the following research question: Can wearables be 
used to identify emotions in everyday life supporting pervasive computing? To refine the number of studies considered in our SLR, we support our question with a set of criteria.

Inclusion criteria:

- Various emotions identified.

- Personal device/wearable used.

- At least one physiological signal monitored.

Exclusion criteria:

- Study performed on a population smaller than five subjects.

- Single emotion or its levels is considered.

- Device is not personal/wearable/portable.

- Device or its modules are connected with a cable.

We used three databases to find articles relevant to our research question: Scopus, Web of Science, and Google Scholar via Publish or Perish. Our search was narrowed down by the following terms: emotion, affective, wearable, smartwatch, smart device, smart band, personal device, iot, ambient intelligence. In total, 2,424 papers were found. At the current stage, $1,104(45 \%)$ articles were carefully reviewed. Only 27 of them $(2.5 \%)$ satisfied our research criteria. We have prioritized papers including emotion recognition phrase in the title or abstract; therefore, it is likely that the majority of the relevant papers are already identified at this stage.

\section{General Study Design for Emotion Detection}

We can distinguish six stages in research design for emotion recognition for pervasive computing, Fig. 1. Decision about emotional model directly impact on reasoning output, Sec. IV-A The second stage covers recruitment, training, profiling and selection of study participants. Some of them need to be excluded due to diseases, e.g. heart problems can significantly interfere with physiological ECG or PPG signals. Then, the appropriate study setup has to be planned differently for a laboratory and field environment (pervasive computing). For laboratory studies, stimuli with annotations may be prepared, whereas ecological momentary assessment (EMA) questionnaires are more suitable for field studies. Next, physiological signals from wearables are collected (Sec. IV-C) together with self-assessments forms. Raw signals are pre-processed, sampled, synchronized and descriptive features are derived, Sec. IV-F, Fig. 2. The selected features combined with the ground truth emotional labels are used to train reasoning model, Sec. IV-G. To adjust the model, hyper-parameter optimization should be considered in order to make it ready for ubiquitous computing.

\section{CRucial Research COMPONEnTs}

\section{A. Emotional Models}

Since human beings are complex, also their emotions can be modelled using various concepts. There is no single, commonly agreed emotion model. In general, categorical and multidimensional approaches are utilized. The former distinguishes several discrete emotional categories like six types of emotions proposed by Ekman and Friesen [14] used in

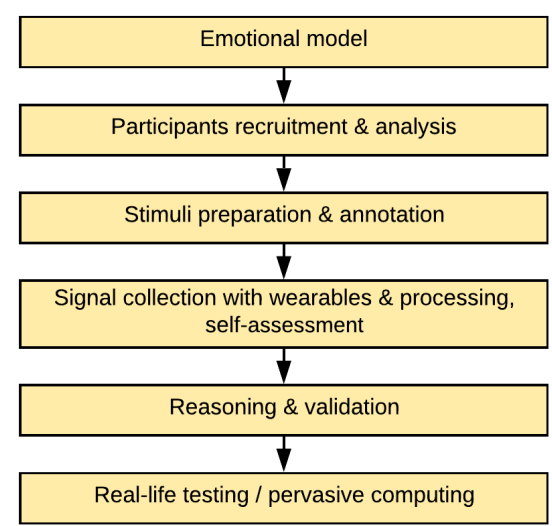

Fig. 1. Research stages for emotion recognition in the real-life environment.

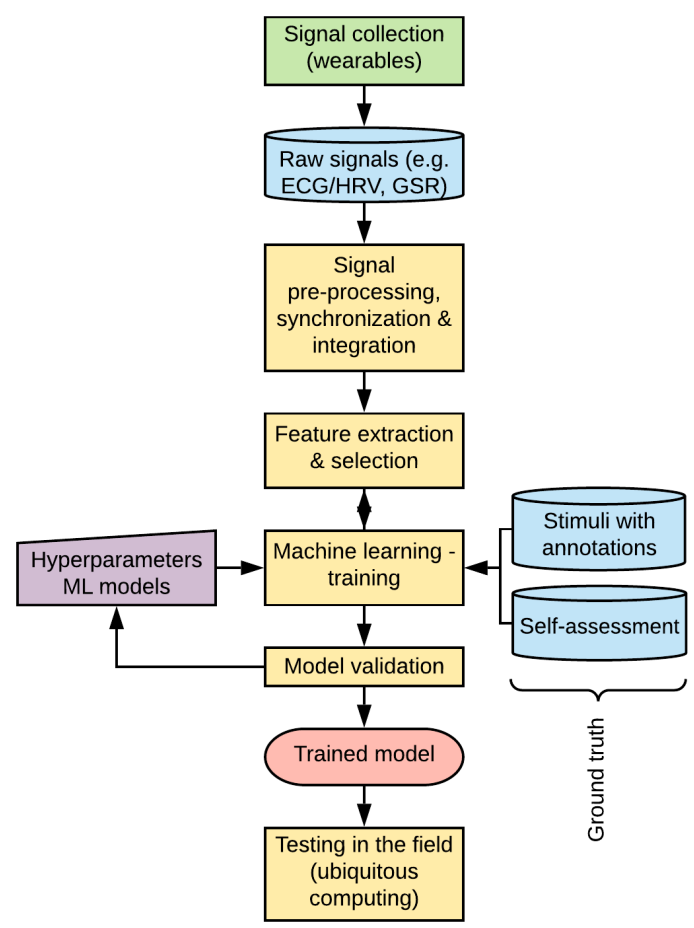

Fig. 2. Emotion recognition process using biological signals from wearables.

[15]; Plutchik's 'wheel of emotions' [16] with 8 primary emotions utilized as $8 \times 3=24$ emojis [17] (only four+neutral were actually analyzed more in-depth) or limited to only 6 basic emotions [18]. Many authors decided to apply their own emotion categories, usually as a subset or small modification of the classical ones, e.g. happy-sad-anger + neutral state [19] extended in [20] with fear, happy-sad-anger-pain [21] joysadness-anger-pleasure [22], or anger-sadness-fear-disgustjoy/amusement + neutral [23], also extended with the seventh one - affection [24], anger-sadness-fear-surprise-frustrationamusement [25], extended in [26] with disgust-other (they did 
not applied these two to reasoning), excited-bored-stressedrelaxed-happy [27]. The number of reported categorical emotions can be even greater like as many as eight+neutral in [28]. In [29], happy-sad-flow categories reflecting flow theory were used. Sometimes, authors treated each emotion as an independent but potentially co-occurring state, each having its level/scale [26], [30]. It actually makes them a multidimensional approach.

A multi-dimensional emotion model assumes multiply dimensions and separate values assigned to each. The most popular is the 2-dimensional arousal-valence Russell's model. Arousal denotes affective intensity and valence emotion type (between sad and happy). Typically, dimension values are discrete, e.g. binary states low-high [28], [31] (forming four quadrants in the 2-dimensional space) or 3-valued lowmedium/neutral-high each [32], [33]. They can be transferred into discrete classes, e.g. joy-sad-stress-calm [31], [34], happybored-neutral [7]. Sometimes, the third dimension: dominance [35], liking [36] or relaxed [6] is also used. If only valence is applied, we receive a simple binary model like negative/fear/sad against positive/relax/happy [37], sometimes extended with neutral [38]. Single valence may have multiple levels [38], [39].

There are also categorical approaches, which combine stress with some other states like neutral-stress-amusement [5] or with more other states [27].

\section{B. Participants}

There are significant differences in experiencing emotions between individuals. A several studies revealed that the age [40], gender [41], and personality [42] have influence on emotions. In many papers the health of the participants is considered, especially a treatment history and current medications [5], [6], [17], [24], [30], [31], [35], information about vision correction [24], [33], and pregnancy [5]. Some experiments are preceded with a questionnaire about depression [22], [24], excessive sweating [17], or the use of cigarettes/tobacco, alcohol or coffee [5], [31], [33], [35]. The total number of participants enrolled vary from two up to nearly two hundred. Some authors disclose whether the study participants volunteered [24], or received a gratification [33].

Surprisingly, only a few studies mention they are approved by the ethical committee [17], [22], [24], [38], [39].

\section{Signals and Sensors}

Detecting emotions from the physiological state requires monitoring body condition by tracking parameters such as heart rate (HR), galvanic skin response (GSR), body temperature (BT). SLR revealed the following signals (Tab. I) are used for detecting affect for pervasive computing. Electrocardiography (ECG) measures the electrical activity of the heart, photoplethysmogram (PPG) registers flow changes in blood volume of the monitored vessel, GSR quantifies variation in the skin conductance. Electroencephalography (EEG) records the electrical activity of the brain, and respiration (RSP) is the breathing rate. Additional set of parameters (Tab. II) is derived from the above mentioned. HR is the number of heartbeats per minute, while heart rate variability (HRV) describes the variation between interbeat intervals (IBI).

TABLE I

PHYSIOLOGICAL SIGNALS USED FOR EMOTION RECOGNITION

\begin{tabular}{|c|c|}
\hline Signal & Used by \\
\hline ECG & [18], |20|, |22], |23], |30], |36] \\
\hline PPG & $|24|,|27|$ \\
\hline $\begin{array}{l}\text { GSR, EDA, EDR, } \\
\text { SC }\end{array}$ & 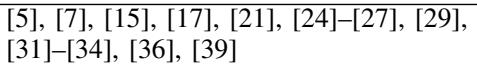 \\
\hline EEG & $|19|,|28|,|35|-|38|$ \\
\hline RSP & 22 \\
\hline BT & [5], โ15], |21], |25]-|27], |29], |31], |39] \\
\hline
\end{tabular}

TABLE II

PARAMETERS DERIVED FROM PHYSIOLOGICAL SIGNALS

\begin{tabular}{|c|c|}
\hline Parameter & Used by \\
\hline HR & $[6],|17|, \mid 21], \mid 25], \mid 26],|29|, \mid 33],|39|$ \\
\hline HRV & $|\overline{2} 9|,|\overline{3} 0|, \overline{3} 4 \mid$ \\
\hline IBI & [6], $]$ \\
\hline BVP & $|\overline{5}|,|\overline{15}|,|28,| 31,], \mid 32]$ \\
\hline
\end{tabular}

We noted several commercially available wearable devices, that seem to be comfortable and useful for collecting physiological signals throughout the day, see Tab. III In addition, some articles propose self-made devices [18], [20], [22], [23].

TABLE III

WEARABLES FOR COLLECTING PHYSIOLOGICAL SIGNALS

\begin{tabular}{|c|c|c|}
\hline Device & Sensors & Used by \\
\hline Empatica E4 & $\begin{array}{l}\text { PPG, GSR, } \\
\text { BT }\end{array}$ & $\frac{|5|, \mid 15],|17|, \mid 21],}{|27|-|29|,|31|-|33|}$ \\
\hline Microsoft Band 2 & PPG, GSR, & $\mid 32],|34|, \mid 39]$ \\
\hline Samsung Gear S & $\mathrm{HR}$ & $|6|$ \\
\hline $\begin{array}{l}\text { BodyMedia SenseWear } \\
\text { Armband }\end{array}$ & $\begin{array}{l}\text { HR, GSR, } \\
\text { BT }\end{array}$ & $|25|,|26|$ \\
\hline Neurosky MindWave & EEG & $\mid 19],|36|-|38|$ \\
\hline XYZlife Bio-Clothing & ECG & $|30|$ \\
\hline
\end{tabular}

\section{Emotion Self-assessment}

A big challenge for studies on human emotions is to obtain ground truth. Authors most commonly request subjects to selfreport their emotional state. However, no information or not enough details about self-assessment are the issue in seven studies [7], [20], [22], [24], [32], [34], [37].

Authors usually ask about the intensity of the experienced emotions in Likert scale (five papers) [6], [30], [31], [36], [38] or the subjects select their emotions from the closed list (three cases) [15], [27], [28]. In [19], the open-ended questions are used, and in two other papers, the combination of open-ended and close-ended questions is applied [25], [26].

Some researchers use the well-established questionnaires or their modified versions. In six studies, Self Assessment Manikin (SAM) is applied [5], [15], [21], [33], [35], [39]. Much less frequently, the IAPS qustionare is utilized [18], [23], AniAvatare [17], Photographic Affect Meter (PAM) [15], State-Trait Anxiety Inventory (STAI) [15], Game Experience Questionnaire (GEQ) [29], Positive and Negative Affect Schedule (PANAS) [5], and Short Stress State Questionnaire [5]. In [7], adult experts assess emotional state based on children face expression. 


\section{E. Stimuli}

There are many procedures to elicit emotions. Most often used are affective videos [5], [17], [21], [22], [24], [25], [28], [30], [31], [38], images [18], [23], [32], [33], [37], and music/sounds [19], [22], [32], [36], [43]. They are easy to explain, simple to apply and available in many data sets: IAPS [44], NAPS [45], CAPS [46], IADS [47], MAHNOB [48].

On the other hand, many studies use stimuli that can be experienced in real-life like playing a game [29], [32], solving a math problem [25], learning [27], walking around the city [39], and other [6], [7], [15], [49]. However, most of the experiments were performed in the lab, where obtaining highquality measurements is relatively easy, and only a few studies were conducted in an uncontrolled environment [6], [15], [17], [39].

Usually, the exposure to stimuli lasts 5-10 seconds per image, 15-30 seconds per audio, 2-5 minutes per video, and 2-60 minutes in case of everyday activities.

\section{F. Signal Processing}

Most of the papers follow the classical approach to signal processing in the field of machine learning, consisting of three explicit stages: pre-processing, feature extraction and selection, Fig. 2. The role of pre-processing is to remove from signals information that is not related to emotional patterns and can negatively impact on results. It also helps to extract the discriminative features more efficiently. The most frequently used pre-processing methods are gathered in Table IV

\section{TABLE IV}

THE MOST POPULAR METHODS USED FOR PRE-PROCESSING

\begin{tabular}{|l|l|}
\hline Pre-processing method & Used by \\
\hline $\begin{array}{l}\text { Filtration (lowpass, bandpass, notch, } \\
\text { median, drift removal) }\end{array}$ & {$[5],[7],[15],[20],[24]$,} \\
\hline $\begin{array}{l}\text { Normalization (to } \pm 1 \text { or [0,1] range) and } \\
\text { standardization (dividing by SD) }\end{array}$ & $[27],[28], \mid 30],[31],[35]$ \\
\hline $\begin{array}{l}\text { Winsonization (removing outliers and } \\
\text { dubious or corrupted fragments, } \\
\text { interpolation of removed samples) }\end{array}$ & {$[7],[22],[34],[37]$} \\
\hline
\end{tabular}

The stage of feature extraction is used to reduce the dimensionality of the problem while maintaining the relevant information. It results in the feature vector representing the original signal or its segment. Usually, features are calculated in time or other domain Tab. V, i.e. an original variable is transformed or decomposed into other signals. Then, different metrics are superimposed on them.

The last stage, consisting in the selection of a subset of features, focuses on the further reduction of dimensionality, taking into account the redundancy of previously extracted features or their inability to distinguish between considered classes. Carrying it out increases the efficiency of classification algorithms. Despite this, the feature selection is not mentioned or performed in most of the reviewed papers. Only in [22] a genetic algorithm is used, and in [31] Sequential Forward Floating Selection is applied. Two more works perform Principal Component Analysis (PCA) [7], [31].

\section{G. Reasoning Models}

Only four studies apply a deep learning algorithm such as convolutional neural networks [29], [39], long short-term memory neural networks [28], [39], or neural networks with backpropagation [25]. All other works use simple supervised learning models among which Support Vector Machine is the most often used.

TABLE V

THE MOST POPULAR METHODS USED FOR FEATURE EXTRACTION

\begin{tabular}{|c|c|c|}
\hline \multicolumn{2}{|c|}{ Feature extraction method } & Used by \\
\hline \multirow{3}{*}{ Time domain } & $\begin{array}{l}\text { Signal morphology } \\
\text { (amplitude, extrema, } \\
\text { intervals, etc.) }\end{array}$ & $\begin{array}{l}|5|,|6|, \mid 20]-\mid 26], \\
\mid 29], \mid 32], \mid 35]\end{array}$ \\
\hline & Rate of specific events & $\begin{array}{l}|5|,|6|, \mid 22], \mid 23], \\
|30|,|33|\end{array}$ \\
\hline & RMS & $[18], \mid 24],[32]$ \\
\hline \multirow{2}{*}{$\begin{array}{l}\text { Frequency } \\
\text { domain }\end{array}$} & PSD & $|5|,|28|, \mid 31],|33|$, \\
\hline & Frequency spectrum & $[28|| 30],, \mid 31], \mid 36]$ \\
\hline $\begin{array}{l}\text { Time-frequency } \\
\text { or time-scale } \\
\text { domain }\end{array}$ & $\begin{array}{l}\text { STFT, WT (CWT, DWT, } \\
\text { FWT) }\end{array}$ & $|7|, \mid 18], \mid 35],|38|$ \\
\hline $\begin{array}{l}\text { Statistical } \\
\text { indices }\end{array}$ & $\begin{array}{l}\text { Mean, median, SD, } \\
\text { skewness, kurtosis, etc. }\end{array}$ & $\begin{array}{l}|5|-|7|,|18|, \\
|22|-|26|,|28|, \\
|30|-|36|\end{array}$ \\
\hline $\begin{array}{l}\text { Nonlinear } \\
\text { measures }\end{array}$ & $\begin{array}{l}\text { Measures of chaos, } \\
\text { complexity, entropy }\end{array}$ & {$[28],[31],[35]$} \\
\hline
\end{tabular}

Really surprising is the fact that only two papers mention imbalance in collected samples [29], [36]. When dealing with multiple emotions (or even just two) it is very unlikely that the distribution will be equal, i.e. each emotion will occur a similar number of times. At the same time, the majority of works use the accuracy as the classification quality measure, which is not appropriate for imbalanced data sets (unless calculated for the correctly classified cases only).

Furthermore, most papers do not provide information on model validation, in particular, evaluated hyper-parameters. Just three compare diverse setups [7], [22], [28], two examine different feature sets [23], [31], and a few test various algorithms [6], [23], [25], [26], [31], [32], [35]. Only [39] addresses a vital question whether to train a general model for all subjects or to build multiple personalized classifiers adjusted to each individual.

The most common validation techniques are $\mathrm{k}$-fold and leave-one-out cross-validation. Labels (output and ground truth for models) are usually obtained from self-assessments or annotated stimuli data. A less popular approach is to employ experts (psychologists) [7], [36], [38].

\section{Applications to Pervasive Computing And CONTEXT-AWARE SYSTEMS}

Affect recognition presents a wide range of possible applications in pervasive computing. With progressive advancement in physiological sensor signal quality and miniaturization, use cases including emotion detection are at the fingertips. During SLR, we have noticed two main application areas emerging: human-computer interaction (HCI) and healthcare.

HCI can be greatly improved once the computer understands human affect [34]. An affective loop can be established to learn 
and foster user experience, and virtual assistants may respond better [32]. Recommendations for search engine results [36], user interface and content [25], [26] may be enhanced with user's emotional context. Affect-aware robots might provide better user experience [18], [23], and television content suggestions could be more accurate [38]. Emotions play an important role in computer games, [32] supporting game-world design process based on a player's affective model. Player's experience can be crafted to emotional feedback [35], making it more realistic.

Healthcare can benefit from the fact that the physiological system and ability to feel are intertwined. Ubiquitous emotion detection can aid to monitor our well-being [20], [24]. Furthermore, emotion recognition could help at stress control, e.g. in order to reduce the probability of cardiovascular diseases [5]. Mental health could be monitored by means of affect detection [6], [30], [39]; emotional self-awareness can improve mental health [17] In [31], the authors alert that negative emotional states can have a degrading impact on our health. Therapeutic contexts are proposed in [7], [21], where children with autism could be taught to understand their emotions better. Affect recognition is also proposed as a comprehensive tool for helping people with emotion-based disorders [22].

Other applications include: detecting driver drowsiness and establishing cognitive load [28], monitoring classroom attitude [27], lesson content difficulty adjustment [29], and creating alternative emotional channel via music [19].

\section{DisCUSSION}

Simple emotional models like low arousal-high arousal or no stress-low stress-high stress were extensively explored. It mainly resulted from the strong correlation between arousal or stress and some biological signals - GSR or BVP [4], [50]. However, emotions are much more complex [51], and their multidimensional nature remains a great challenge for future work. This also appears to be the main reason why in only a few studies, the authors monitor and recognize emotions in the non-controlled (field study) [6], [15], [39] or semi-controlled environment, e.g. emotion monitoring while watching the live football game in TV [17].

Emotion model directly impacts on the detection model. Various emotion models make it almost impossible to compare results with each other, e.g. does fear from [20] corresponds to pain in [21]? Multi-dimensional models should straightforward lead to a multi-label classification problem, which in turn requires much more cases to train the classifier. None of the paper approach to reasoning in this way. Moreover, correct multi-label models can recognize combinations that do not occur in the training set.

There is actually no research, which could be seen as comprehensive with respect to all research design stages, Fig. 1. For example, only two out of all 27 papers consider and properly solve the imbalanced data problem, three optimize machine learning model parameters, five claim to have an ethical committee approval, another five recruited more than 50 participants, sampling rates of the sensor signals are commonly not considered at all. Finally, only four identify complex emotions in real-life conditions.

We focused on pervasive wireless wearables that can monitor physiological signals. However, some other signals and data may be complementary, e.g. data gathered by our smartphone about our activity [52], voice [53], or smartwatch builtin camera monitoring our face [54].

The low variety of hardware tested might stem from the fact that most off-the-shelf wearables do not provide access to raw physiological signals.

\section{CONCLUSIONS}

In our systematic literature review (SLR), we analyzed methods and solutions that enable us to effectively recognize human emotions in everyday life and utilize such information in pervasive computing. Half-way through, based on the literature, we believe that such a solution is achievable but still requires further investigation.

There are still many challenges and problems to solve in order to ensure high quality of emotion detection in contextaware applications. We found a great potential, especially in the improvement of signal data processing, model learning, and tailoring the suitable deep machine learning architecture.

We have shown that emotion recognition from personal devices is already a mature but very promising direction for further research.

\section{REFERENCES}

[1] J. S. Lerner, Y. Li, P. Valdesolo, and K. S. Kassam, "Emotion and decision making," Annual review of psychology, vol. 66, pp. 799-823, 2015.

[2] D. Kahneman, Thinking, fast and slow. Macmillan, 2011.

[3] C. A. Smith, R. S. Lazarus et al., "Emotion and adaptation," Handbook of personality: Theory and research, pp. 609-637, 1990.

[4] P. Schmidt, A. Reiss, R. Dürichen, and K. V. Laerhoven, "Wearablebased affect recognition-a review," Sensors, vol. 19, no. 19, p. 4079, 2019.

[5] P. Schmidt, A. Reiss, R. Duerichen, C. Marberger, and K. Van Laerhoven, "Introducing wesad, a multimodal dataset for wearable stress and affect detection," in Proceedings of the 2018 on International Conference on Multimodal Interaction. ACM, 2018, pp. 400-408.

[6] M. R. Kamdar and M. J. Wu, "Prism: a data-driven platform for monitoring mental health," in Biocomputing 2016: Proceedings of the Pacific Symposium. World Scientific, 2016, pp. 333-344.

[7] H. Feng, H. M. Golshan, and M. H. Mahoor, "A wavelet-based approach to emotion classification using eda signals," Expert Systems with Applications, vol. 112, pp. 77-86, 2018.

[8] M. Z. Soroush, K. Maghooli, S. K. Setarehdan, and A. M. Nasrabadi, "A review on eeg signals based emotion recognition," International Clinical Neuroscience Journal, vol. 4, no. 4, p. 118, 2017.

[9] T. Xu, Y. Zhou, Z. Wang, and Y. Peng, "Learning emotions eeg-based recognition and brain activity: A survey study on bci for intelligent tutoring system," Procedia computer science, vol. 130, pp. 376-382, 2018.

[10] C. Marechal, D. Mikołajewski, K. Tyburek, P. Prokopowicz, L. Bougueroua, C. Ancourt, and K. Węgrzyn-Wolska, "Survey on ai-based multimodal methods for emotion detection," in High-Performance Modelling and Simulation for Big Data Applications. Springer, 2019, pp. 307-324.

[11] E. Maria, L. Matthias, and H. Sten, "Emotion recognition from physiological signal analysis: A review," Electronic Notes in Theoretical Computer Science, vol. 343, pp. 35-55, 2019.

[12] L. Shu, J. Xie, M. Yang, Z. Li, Z. Li, D. Liao, X. Xu, and X. Yang, "A review of emotion recognition using physiological signals," Sensors, vol. 18, no. 7, p. 2074, 2018. 
[13] B. Kitchenham, "Procedures for undertaking systematic reviews: Joint technical report," Computer Science Department, Keele University (TR/SE-0401) and National ICT Australia Ltd.(0400011T. 1), 2004.

[14] P. Ekman and W. V. Friesen, "Facial Action Coding System: A technique for the measurement of facial movement, Consulting Psychologists Press," Palo Alto, CA, 1978.

[15] P. Schmidt, A. Reiss, R. Dürichen, and K. Van Laerhoven, "Labelling affective states in the wild: Practical guidelines and lessons learned," in Proceedings of the 2018 ACM International Joint Conference and 2018 International Symposium on Pervasive and Ubiquitous Computing and Wearable Computers. ACM, 2018, pp. 654-659.

[16] R. Plutchik, Emotions and life: Perspectives from psychology, biology, and evolution. American Psychological Association, 2003.

[17] A. Albraikan, B. Hafidh, and A. El Saddik, "iaware: A real-time emotional biofeedback system based on physiological signals," IEEE Access, vol. 6, pp. 78780-78 789, 2018.

[18] K. Rattanyu, M. Ohkura, and M. Mizukawa, "Emotion monitoring from physiological signals for service robots in the living space," in ICCAS 2010. IEEE, 2010, pp. 580-583.

[19] X. Lu, X. Liu, and E. Stolterman Bergqvist, "It sounds like she is sad: Introducing a biosensing prototype that transforms emotions into realtime music and facilitates social interaction," in Extended Abstracts of the 2019 CHI Conference on Human Factors in Computing Systems. ACM, 2019, p. LBW2219.

[20] L. Hu, J. Yang, M. Chen, Y. Qian, and J. J. Rodrigues, "Scai-svsc: Smart clothing for effective interaction with a sustainable vital sign collection," Future Generation Computer Systems, vol. 86, pp. 329-338, 2018.

[21] D. Pollreisz and N. TaheriNejad, "A simple algorithm for emotion recognition, using physiological signals of a smart watch," in 2017 39th Annual International Conference of the IEEE Engineering in Medicine and Biology Society (EMBC). IEEE, 2017, pp. 2353-2356.

[22] C. He, Y.-j. Yao, and X.-s. Ye, "An emotion recognition system based on physiological signals obtained by wearable sensors," in Wearable sensors and robots. Springer, 2017, pp. 15-25.

[23] K. Rattanyu and M. Mizukawa, "Emotion recognition using biological signal in intelligent space," in International Conference on HumanComputer Interaction. Springer, 2011, pp. 586-592.

[24] L. Fernández-Aguilar, A. Martínez-Rodrigo, J. Moncho-Bogani, A. Fernández-Caballero, and J. M. Latorre, "Emotion detection in aging adults through continuous monitoring of electro-dermal activity and heart-rate variability," in International Work-Conference on the Interplay Between Natural and Artificial Computation. Springer, 2019, pp. 252261.

[25] C. L. Lisetti and F. Nasoz, "Categorizing autonomic nervous system (ans) emotional signals using bio-sensors for hri within the maui paradigm," in ROMAN 2006-The 15th IEEE International Symposium on Robot and Human Interactive Communication. IEEE, 2006, pp. 277-284.

[26] C. L. Lisetti and F. Nasoz, "Using noninvasive wearable computers to recognize human emotions from physiological signals," EURASIP journal on applied signal processing, vol. 2004, pp. 1672-1687, 2004.

[27] M. S. Dao, D. Nguyen, D. Tien, A. Kasem et al., "Healthyclassrooma proof-of-concept study for discovering students' daily moods and classroom emotions to enhance a learning-teaching process using heterogeneous sensors," 2018.

[28] B. Nakisa, M. N. Rastgoo, A. Rakotonirainy, F. Maire, and V. Chandran, "Long short term memory hyperparameter optimization for a neural network based emotion recognition framework," IEEE Access, vol. 6 , pp. 49325-49338, 2018

[29] M. Maier, C. Marouane, and D. Elsner, "Deepflow: Detecting optimal user experience from physiological data using deep neural networks," in Proceedings of the 18th International Conference on Autonomous Agents and MultiAgent Systems. International Foundation for Autonomous Agents and Multiagent Systems, 2019, pp. 2108-2110.

[30] H. W. Guo, Y. S. Huang, J. C. Chien, and J. S. Shieh, "Short-term analysis of heart rate variability for emotion recognition via a wearable ecg device," in 2015 International Conference on Intelligent Informatics and Biomedical Sciences (ICIIBMS). IEEE, 2015, pp. 262-265.

[31] B. Zhao, Z. Wang, Z. Yu, and B. Guo, "Emotionsense: Emotion recognition based on wearable wristband," in 2018 IEEE SmartWorld, Ubiquitous Intelligence \& Computing, Advanced \& Trusted Computing, Scalable Computing \& Communications, Cloud \& Big Data Computing, Internet of People and Smart City Innovation (Smart-
World/SCALCOM/UIC/ATC/CBDCom/IOP/SCI) . IEEE, 2018, pp. 346355.

[32] G. J. Nalepa, K. Kutt, B. Giżycka, P. Jemioło, and S. Bobek, "Analysis and use of the emotional context with wearable devices for games and intelligent assistants," Sensors, vol. 19, no. 11, p. 2509, 2019.

[33] M. Ragot, N. Martin, S. Em, N. Pallamin, and J.-M. Diverrez, "Emotion recognition using physiological signals: laboratory vs. wearable sensors," in International Conference on Applied Human Factors and Ergonomics. Springer, 2017, pp. 15-22.

[34] F. Setiawan, S. A. Khowaja, A. G. Prabono, B. N. Yahya, and S.-L. Lee, "A framework for real time emotion recognition based on human ans using pervasive device," in 2018 IEEE 42nd Annual Computer Software and Applications Conference (COMPSAC), vol. 1. IEEE, 2018, pp. 805-806.

[35] T. Xu, R. Yin, L. Shu, and X. Xu, "Emotion recognition using frontal eeg in vr affective scenes," in 2019 IEEE MTT-S International Microwave Biomedical Conference (IMBioC), vol. 1. IEEE, 2019, pp. 1-4.

[36] R. Gupta, M. Khomami Abadi, J. A. Cárdenes Cabré, F. Morreale, T. H. Falk, and N. Sebe, "A quality adaptive multimodal affect recognition system for user-centric multimedia indexing," in Proceedings of the 2016 ACM on international conference on multimedia retrieval. ACM, 2016, pp. 317-320.

[37] Y. Nie, Y. Wu, Z. Yang, G. Sun, Y. Yang, and X. Hong, "Emotional evaluation based on svm," in 2017 2nd International Conference on Automation, Mechanical Control and Computational Engineering (AMCCE 2017). Atlantis Press, 2017.

[38] A. Jalilifard, A. G. da Silva, and M. K. Islam, "Brain-tv connection: Toward establishing emotional connection with smart tvs," in 2017 IEEE Region 10 Humanitarian Technology Conference (R10-HTC). IEEE, 2017, pp. 726-729.

[39] E. Kanjo, E. M. Younis, and C. S. Ang, "Deep learning analysis of mobile physiological, environmental and location sensor data for emotion detection," Information Fusion, vol. 49, pp. 46-56, 2019.

[40] L. Fernández-Aguilar, J. Ricarte, L. Ros, and J. M. Latorre, "Emotional differences in young and older adults: Films as mood induction procedure," Frontiers in psychology, vol. 9, p. 1110, 2018.

[41] Y. Deng, L. Chang, M. Yang, M. Huo, and R. Zhou, "Gender differences in emotional response: Inconsistency between experience and expressivity," PloS one, vol. 11, no. 6, p. e0158666, 2016.

[42] A. Costa, J. A. Rincon, C. Carrascosa, V. Julian, and P. Novais, "Emotions detection on an ambient intelligent system using wearable devices," Future Generation Computer Systems, vol. 92, pp. 479-489, 2019.

[43] M. Magno, M. Pritz, P. Mayer, and L. Benini, "Deepemote: Towards multi-layer neural networks in a low power wearable multi-sensors bracelet," in 2017 7th IEEE International Workshop on Advances in Sensors and Interfaces (IWASI). IEEE, 2017, pp. 32-37.

[44] P. J. Lang, "International affective picture system (iaps): Affective ratings of pictures and instruction manual," Technical report, 2005.

[45] A. Marchewka, Ł. Żurawski, K. Jednoróg, and A. Grabowska, "The nencki affective picture system (naps): Introduction to a novel, standardized, wide-range, high-quality, realistic picture database," Behavior research methods, vol. 46, no. 2, pp. 596-610, 2014.

[46] B. Lu, M. Hui, and H. Yu-Xia, "The development of native chinese affective picture system-a pretest in 46 college students." Chinese Mental Health Journal, 2005.

[47] M. M. Bradley and P. J. Lang, "The international affective digitized sounds (; iads-2): Affective ratings of sounds and instruction manual," University of Florida, Gainesville, FL, Tech. Rep. B-3, 2007.

[48] M. Soleymani, J. Lichtenauer, T. Pun, and M. Pantic, "A multimodal database for affect recognition and implicit tagging," IEEE Transactions on Affective Computing, vol. 3, no. 1, pp. 42-55, 2011.

[49] M. V. Sokolova, A. Fernández-Caballero, M. T. López, A. MartínezRodrigo, R. Zangróniz, and J. M. Pastor, "A distributed architecture for multimodal emotion identification," in Trends in Practical Applications of Agents, Multi-Agent Systems and Sustainability. Springer, 2015, pp. $125-132$

[50] J. Choi, B. Ahmed, and R. Gutierrez-Osuna, "Development and evaluation of an ambulatory stress monitor based on wearable sensors," IEEE transactions on information technology in biomedicine, vol. 16, no. 2 , pp. 279-286, 2011.

[51] A. S. Cowen and D. Keltner, "Self-report captures 27 distinct categories of emotion bridged by continuous gradients," Proceedings of the National Academy of Sciences, vol. 114, no. 38, pp. E7900-E7909, 2017. 
[52] W. Sasaki, J. Nakazawa, and T. Okoshi, "Comparing esm timings for emotional estimation model with fine temporal granularity," in Proceedings of the 2018 ACM International Joint Conference and 2018 International Symposium on Pervasive and Ubiquitous Computing and Wearable Computers. ACM, 2018, pp. 722-725.

[53] P. Denman, E. Lewis, S. Prasad, J. Healey, H. Syed, and L. Nachman, "Affsens: a mobile platform for capturing affect in context," in Proceedings of the 20th International Conference on Human-Computer Interaction with Mobile Devices and Services Adjunct. ACM, 2018, pp. 321-326.

[54] J. A. Rincon, A. Costa, P. Novais, V. Julian, and C. Carrascosa "Intelligent wristbands for the automatic detection of emotional states for the elderly," in International Conference on Intelligent Data Engineering and Automated Learning. Springer, 2018, pp. 520-530. 